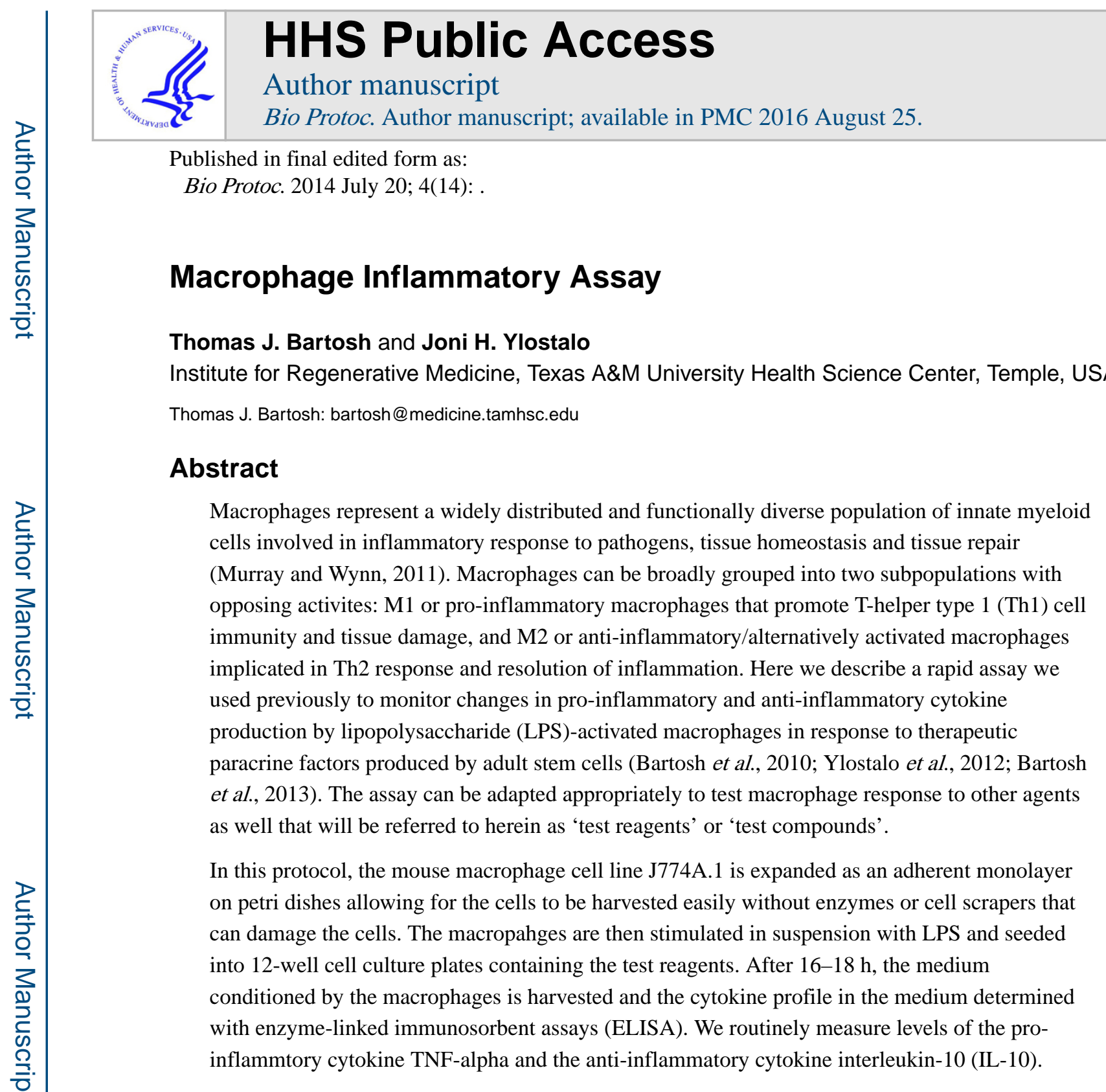

\title{
Materials and Reagents
}

1. J774A.1 mouse macrophages (ATCC, catalog number: TIB-67)

2. $0.1 \mathrm{mg} / \mathrm{ml}$ lipopolysaccharide (LPS) (Sigma-Aldrich, catalog number: L4130) solution in PBS

3. Mouse TNF-alpha Quantikine ELISA kit (R\&D Systems, catalog number: MTA00B)

4. Mouse Interleukin 10 (IL-10) Quantikine ELISA kit (R\&D Systems, catalog number: M1000B)

5. High glucose Dulbecco's modified Eagle medium (DMEM) containing Glutamax (Life Technologies, catalog number: 10569) 
6. Fetal bovine serum (Atlanta Biologicals, catalog number: S11550)

7. Penicillin-streptomycin (Life Technologies, catalog number: 15140)

8. Macrophage medium (see Recipes)

\section{Equipment}

1. $150 \times 15 \mathrm{~mm}$ petri dish (BD Biosciences, Falcon ${ }^{\circledR}$, catalog number: 351058)

2. Stericup-GP $0.22 \mu \mathrm{m}$ vacuum filtration device (EMD Millipore, catalog number: SCGPU05RE)

3. 12-well tissue culture treated plates (Corning, catalog number: 3512)

4. $10 \mathrm{ml}$ capacity serological pipette (VWR International, catalog number: 89130)

5. $\quad 1.5 \mathrm{ml}$ microcentrifuge tubes

6. $\quad 50 \mathrm{ml}$ sterile conical tube (BD Biosciences, Falcon ${ }^{\circledR}$, catalog number: 352070)

7. Water bath set to $37^{\circ} \mathrm{C}$

8. $\quad$ Pipette-aid

9. Centrifuge with swinging-bucket rotor and adaptors for 50-ml conical tubes

10. Humidified cell culture incubator set to $37{ }^{\circ} \mathrm{C}$ and $5 \% \mathrm{CO}_{2}$

11. Upright microscope with $10 \times$ objective

12. Microplate reader (capable of measuring absorbance at $450 \mathrm{~nm}$, with a background correction wavelength of $540 \mathrm{~nm}$ or $570 \mathrm{~nm}$ )

\section{Procedure}

A. Preparation of macrophage cultures

1. Expand the J774A.1 mouse macrophages in macrophage medium on a $15 \mathrm{~cm}$ petri dish.

Notes:

a. A detailed method for culture of mouse macrophages can be found in references below.

b. Macrophage medium should be changed every 2-3 days.

c. The use of petri dishes allows the macrophages to be harvested for the assay without dissociating enzymes and without the 
requirement of a cell scraper that can be damaging to the cells.

2. Upon reaching $70-80 \%$ confluence, aspirate the culture medium from the dish and add $10 \mathrm{ml}$ of fresh macrophage medium.

3. Harvest the macrophages by washing them from the petri dish with the macrophage medium using a pipette-aid and $10 \mathrm{ml}$ serological pipette.

Note: Spray the medium over the macrophages multiple times to dislodge the cells from the dish.

4. Transfer the macrophage suspension from the plate to a $50 \mathrm{ml}$ conical tube. Add an additional $10 \mathrm{ml}$ macrophage medium to the plate and repeat wash step.

5. Pellet the cells by centrifugation at $200-250 \times g$ for 5-7 min.

6. Suspend the macrophages in macrophage medium and count the cells.

Notes:

a. A $15 \mathrm{~cm}$ petri dish containing macrophages at $70 \%$ confluence should yield approximately 15 million macrophages.

b. The macrophages will consistently have high viability when cultured and harvested as described. Therefore, the use of a viability dye such as trypan blue for distinguishing live versus dead cells is not mandatory.

7. Prepare a suspension of the macrophages in macrophage medium at a density of 200,000 cells per ml.

B. Preparation of assay plates (Figure 1)

8. Determine the number of 12 -well cell culture plates necessary to assay samples of interest in triplicate.

9. Transfer the test compound(s) of interest in triplicate to the appropriate wells of a 12-well plate.

Note: We have routinely tested the effects of mesenchymal stem cell conditioned medium, prostaglandins such as PGE2, various pharmacological compounds, neutralizing antibodies and recombinant proteins.

10. Add macrophage medium to all wells containing a test reagent to a final volume of $500 \mu \mathrm{l}$. 
Note: If the volume of the test compound employed is minute, it is advantageous to first add the macrophage medium to the wells and then transfer the test compound of interest (from step B9) to the appropriate wells. The final volume in each well should remain $500 \mu \mathrm{l}$.

11. Add $500 \mu \mathrm{l}$ macrophage medium to 6 additional wells not containing a test reagent.

Note: These wells will provide controls for the assay.

12. Transfer $500 \mu \mathrm{l}$ of the macrophage cell suspension $(100,000$ cells) to each of 3 wells containing $500 \mu \mathrm{l}$ of macrophage medium only (without a test reagent).

Note: These wells will serve as unstimulated macrophage controls (Mac controls, Figure 1).

C. Macrophage stimulation with LPS

13. Stimulate the macrophages by adding a 1:500 dilution of the $0.1 \mathrm{mg} / \mathrm{ml}$ solution of LPS to the remainder of the macrophage suspension. To distribute the LPS, immediately mix the cell suspension by pipetting up and down 10-15 times.

14. Place the cap on the $50 \mathrm{ml}$ tube containing the LPS-stimulated macrophages and incubate the cells in the $50 \mathrm{ml}$ tube for $5 \mathrm{~min}$ at room temperature.

15. Mix the cells again then transfer $500 \mu \mathrm{l}(100,000$ cells $)$ to the appropriate wells containing the test samples. Also, add $500 \mu \mathrm{l}$ of the macrophage suspension to the three remaining wells containing $500 \mu \mathrm{l}$ macrophage medium only (LPS-stimulated macrophage controls) (Figure 1).

16. Rock the plate 3 times to distribute the macrophages evenly across the well and incubate at $37^{\circ} \mathrm{C}$ for up to $24 \mathrm{~h}$. Each well for the assay finally contains 100,000 macrophages in $1.0 \mathrm{ml}$ macrophage medium and $100 \mathrm{ng} / \mathrm{ml}$ LPS (except the three unstimulated control wells).

Note: We routinely incubate the macrophages with LPS for approximately 16-18 h. However, an abundance of macrophage-derived cytokines can be detected in the conditioned medium as early as 4-6 h after LPS stimulation.

D. Collection of conditioned medium for assays of cytokine production

17. Prior to collecting the medium conditioned by the macrophages, observe the cultures to verify the cells were properly activated (Figure 2). 
Note: J774A.1 macrophages stimulated with LPS appear larger, flatter, and more granular than un-stimulated macrophages.

18. Collect the medium conditioned by the macrophages and place it into $1.5 \mathrm{ml}$ microcentrifuge tubes.

19. Centrifuge the samples for $5 \mathrm{~min}$ at $500 \times \mathrm{g}$, room temperature.

20. Transfer the supernatant to new $1.5 \mathrm{ml}$ tubes.

Notes:

a. Although a cell pellet should not be visible, avoid touching the bottom of the tube when removing the supernatant.

b. The samples can be used immediately for ELISA assay or aliquoted and stored at $-80{ }^{\circ} \mathrm{C}$.

21. Vortex the medium samples prior to their use in TNF-alpha and IL-10 ELISA assays. Follow instructions provided by the manufacturer of the ELISA kits. The absorbance of the wells can be read on a microplate reader with a wavelength of 450 $\mathrm{nm}$ and background wavelength correction set at $540 \mathrm{~nm}$ or $570 \mathrm{~nm}$. Representative amounts of TNF-alpha and IL-10 produced by macrophages in response to LPS and a test compound (PGE2) are shown in the graphs below (Figure 3).

\section{Notes}

1. We have routinely used mouse TNF-alpha and IL-10 ELISA kits to monitor the specific activity of mouse macrophages in response to paracrine factors, such as PGE2, produced by mesenchymal stem cells. However, production of other macrophage-derived cytokines is also increased by LPS stimulation including CXCL2/MIP2, MCP-1, IL-6, IL-1 beta, and IL12-p40 (Ylostalo et al., 2012). These cytokines can also be measured by ELISA.

2. When the assay is performed as described above, levels of TNF-alpha in the conditioned medium increases from $10-30 \mathrm{pg} / \mathrm{ml}$ in unstimulated macrophage cultures to $800-1,200 \mathrm{pg} / \mathrm{ml}$ in macrophages stimulated for 16-18 h with LPS. Levels of IL-10 increase only slightly with LPS stimulation. However, IL-10 levels can be markedly enhanced in LPSstimulated macrophage cultures by factors that promote an antiinflammatory macrophage phenotype such as PGE2 (Figure 3).

3. Different preparations of LPS can elicit variations in macrophage cytokine production. The concentration of LPS used in the assay can be adjusted to achieve the desired results. We have routinely used LPS concentrations ranging from $10 \mathrm{ng} / \mathrm{ml}$ to $1 \mu \mathrm{g} / \mathrm{ml}$. 


\title{
Recipes
}

1.

\author{
Macrophage medium (500 ml) \\ High glucose DMEM containing Glutamax, $445 \mathrm{ml}$ \\ Fetal bovine serum, $50 \mathrm{ml}$ \\ Penicillin-streptomycin, $5 \mathrm{ml}$ \\ Filters sterilize $(0.22 \mu \mathrm{m})$ and store up to one month at $4{ }^{\circ} \mathrm{C}$ \\ Prewarm to $37{ }^{\circ} \mathrm{C}$ in water bath immediately prior to use
}

\section{Acknowledgments}

This protocol was adapted from our original work (Bartosh et al., 2013; Ylostalo et al., 2012) that was supported by grants from the NIH (P40RR17447) and Cancer Prevention \& Research Institute of Texas (RP110620) to Darwin J. Prockop, Institute for Regenerative Medicine, Texas A\&M University Health Science Center.

\section{References}

1. Bartosh TJ, Ylostalo JH, Bazhanov N, Kuhlman J, Prockop DJ. Dynamic compaction of human mesenchymal stem/precursor cells into spheres self-activates caspase-dependent IL1 signaling to enhance secretion of modulators of inflammation and immunity (PGE2, TSG6, and STC1). Stem Cells. 2013; 31(11):2443-2456. [PubMed: 23922312]

2. Bartosh TJ, Ylostalo JH, Mohammadipoor A, Bazhanov N, Coble K, Claypool K, Lee RH, Choi H, Prockop DJ. Aggregation of human mesenchymal stromal cells (MSCs) into 3D spheroids enhances their antiinflammatory properties. Proc Natl Acad Sci U S A. 2010; 107(31):13724-13729. [PubMed: 20643923]

3. Murray PJ, Wynn TA. Protective and pathogenic functions of macrophage subsets. Nat Rev Immunol. 2011; 11(11):723-737. [PubMed: 21997792]

4. Ylostalo JH, Bartosh TJ, Coble K, Prockop DJ. Human mesenchymal stem/stromal cells cultured as spheroids are self-activated to produce prostaglandin E2 that directs stimulated macrophages into an anti-inflammatory phenotype. Stem Cells. 2012; 30(10):2283-2296. [PubMed: 22865689] 
1) Mac Control

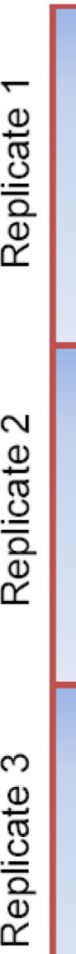

$+$

$500 \mu \mathrm{l} \mathrm{Mac}$

$+$

$500 \mu \mathrm{l} \mathrm{Mac}$
2) LPS-Mac Control

$500 \mu \mathrm{l} \mathrm{Mac}$ medium

$500 \mathrm{l}$ Mac medium

Figure 1. Sample plate layout for the macrophage assay control for the test reagents have been omitted.

To prepare the 12-well assay plate, the test reagents of interest are first added to the appropriate wells in triplicate (shown here in columns 3 and 4). Macrophage (Mac) medium is then added to every well to a final volume of $500 \mu \mathrm{l}$ followed by addition of $500 \mu \mathrm{l}$ of the Mac cell suspension to each well in the first column (unstimulated Mac control). $500 \mu \mathrm{l}$ of the LPS-stimulated macrophages (LPS-Mac) are transferred to each of the remaining 9 wells (columns 2, 3, and 4). Note that three of these wells do not contain a test reagent (shown here in column 2) and therefore serve as the LPS-stimulated macrophage control (LPS-Mac control). The final volume per well is $1.0 \mathrm{ml}$. For simplicity, wells containing a vehicle 

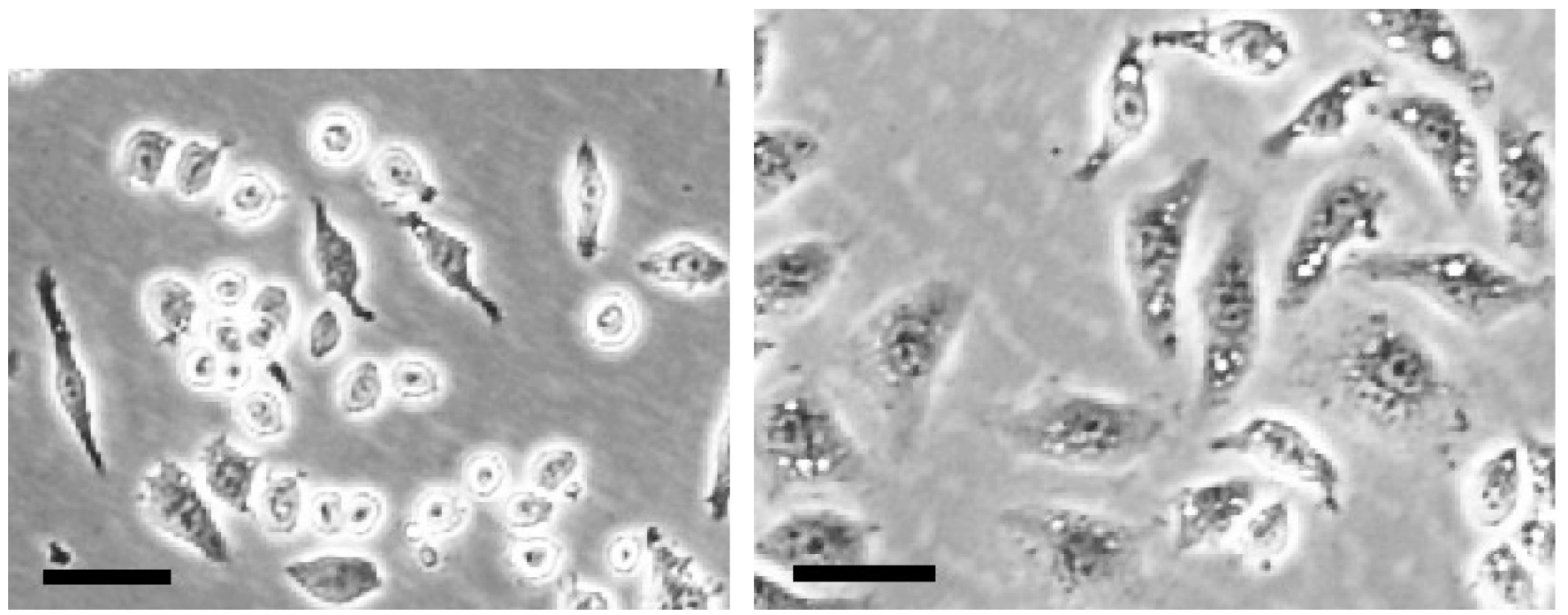

Figure 2. Changes in macrophage morphology in response to LPS

Brightfield images of unsimlulated macrophages (left) and macrophages stimulated with LPS for $18 \mathrm{~h}$ (right). Scale bar $=50 \mu \mathrm{m}$. 

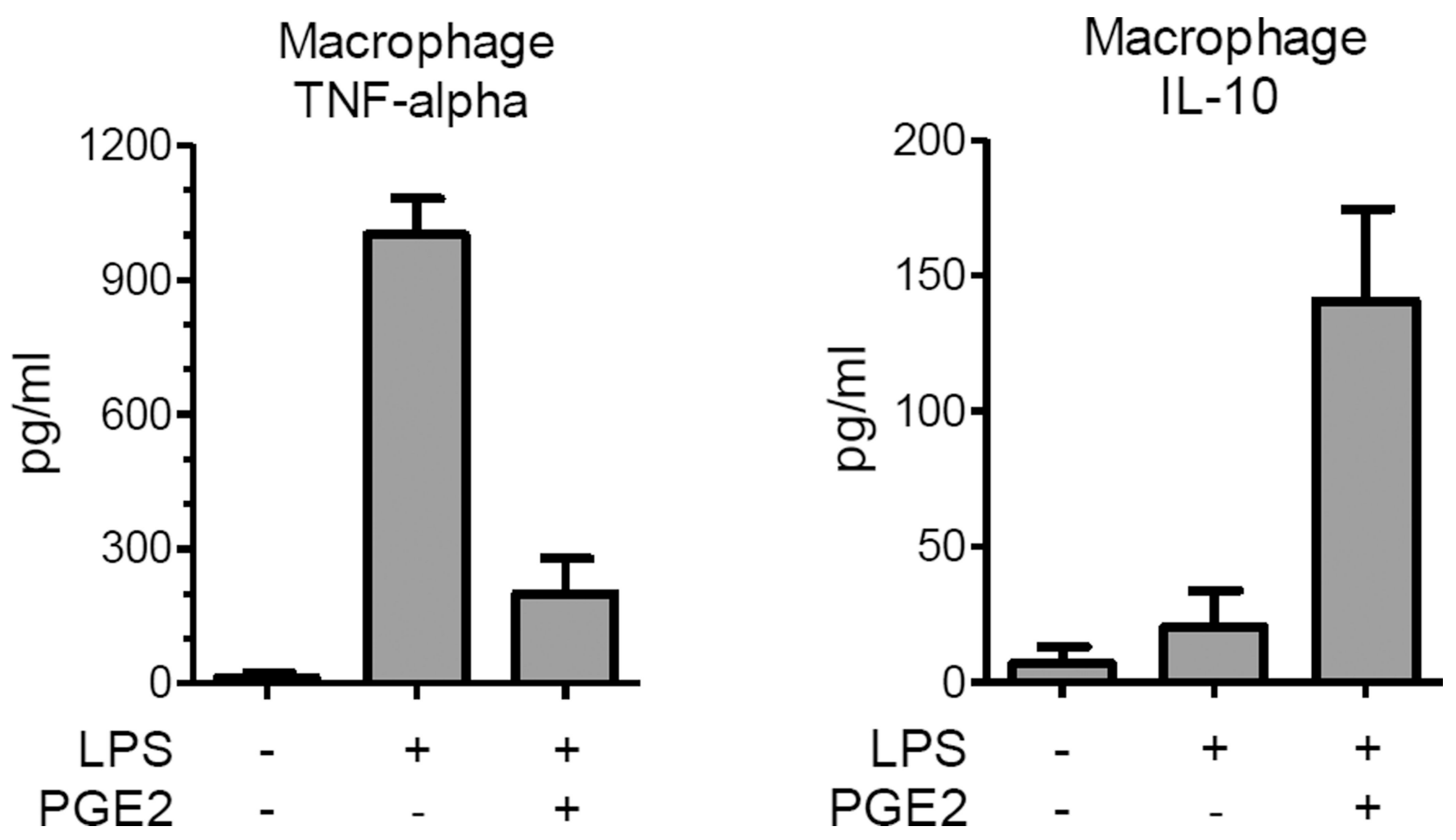

Figure 3. Effects of LPS and PGE2 on TNF-alpha and IL-10 production by macrophages Macrophages were stimulated for $18 \mathrm{~h}$ with $100 \mathrm{ng} / \mathrm{ml}$ LPS alone or LPS in combination with $1 \mathrm{ng} / \mathrm{ml}$ of the immune modulatory factor prostaglandin E2 (PGE2). LPS increases levels of the pro-inflammatory cytokine TNF-alpha produced by the macrophages. PGE2 attenuates macrophage production of TNF-alpha elicited by LPS stimulation but enhances levels of the anti-inflammatory cytokine IL-10. Values are mean $+/-\mathrm{SD}, \mathrm{n}=3$ per group. 